Braz J Med Biol Res, July 2010, Volume 43(7) 645-650

doi: 10.1590/S0100-879X2010007500053

Leukotrienes are not essential for the efficacy of a heterologous vaccine against Mycobacterium tuberculosis infection

L.H. Franco, M. Oliveira e Paula, P.F. Wowk, D.M. da Fonseca, C.A. Sérgio, P.F. Fedatto, A.F. Gembre, S.G. Ramos, C.L. Silva, A.I. Medeiros, L.H. Faccioli and V.L.D. Bonato

The Brazilian Journal of Medical and Biological Research is partially financed by
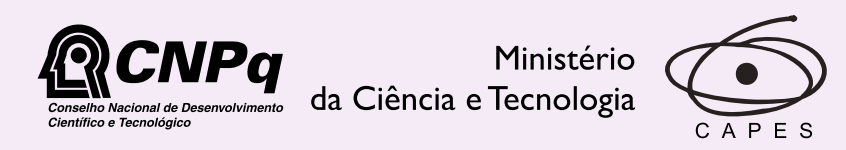
Ministério
da Educação

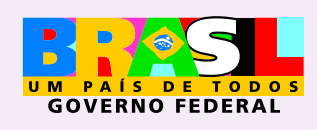

Institutional S ponsors
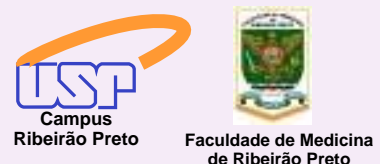


\title{
Leukotrienes are not essential for the efficacy of a heterologous vaccine against Mycobacterium tuberculosis infection
}

\author{
L.H. Franco ${ }^{1}$, M. Oliveira e Paula ${ }^{1}$, P.F. Wowk ${ }^{1}$, D.M. da Fonseca ${ }^{1}$, \\ C.A. Sérgio ${ }^{1}$, P.F. Fedatto ${ }^{1}$, A.F. Gembre1, S.G. Ramos ${ }^{2}$, C.L. Silva ${ }^{1}$, \\ A.I. Medeiros ${ }^{3}$, L.H. Faccioli ${ }^{4}$ and V.L.D. Bonato ${ }^{1}$ \\ ${ }^{1}$ Núcleo de Pesquisas em Tuberculose, Departamento de Bioquímica e Imunologia, \\ 2Departamento de Patologia, Faculdade de Medicina de Ribeirão Preto, \\ Universidade de São Paulo, Ribeirão Preto, SP, Brasil \\ ${ }^{3}$ Departamento de Ciências Biológicas, Faculdade de Ciências Farmacêuticas, \\ Universidade Estadual Paulista, Araraquara, SP, Brasil \\ ${ }^{4}$ Departamento de Análises Clínicas, Toxicológicas e Bromatológicas, \\ Faculdade de Ciências Farmacêuticas de Ribeirão Preto, \\ Universidade de São Paulo, Ribeirão Preto, SP, Brasil
}

\begin{abstract}
Leukotrienes are reported to be potent proinflammatory mediators that play a role in the development of several inflammatory diseases such as asthma, rheumatoid arthritis and periodontal disease. Leukotrienes have also been associated with protection against infectious diseases. However, the role of leukotrienes in Mycobacterium tuberculosis infection is not understood. To answer this question, we studied the role of leukotrienes in the protective immune response conferred by prime-boost heterologous immunization against tuberculosis. We immunized BALB/c mice (4-11/group) with subcutaneous BCG vaccine $\left(1 \times 10^{5} \mathrm{M}\right.$. bovis BCG) (prime) followed by intramuscular DNA-HSP65 vaccine (100 $\mu \mathrm{g})$ (boost). During the 30 days following the challenge, the animals were treated by gavage daily with MK-886 (5 mg $\left.\mathrm{kg}^{-1} \cdot \mathrm{day}^{-1}\right)$ to inhibit leukotriene synthesis. We showed that MK886-treated mice were more susceptible to $M$. tuberculosis infection by counting the number of $M$. tuberculosis colony-forming units in lungs. The histopathological analysis showed an impaired influx of leukocytes to the lungs of MK-886-treated mice after infection, confirming the involvement of leukotrienes in the protective immune response against experimental tuberculosis. However, prime-boost-immunized mice treated with MK-886 remained protected after challenge with $M$. tuberculosis, suggesting that leukotrienes are not required for the protective effect elicited by immunization. Protection against $M$. tuberculosis challenge achieved by prime-boost immunization in the absence of leukotrienes was accompanied by an increase in IL-17 production in the lungs of these animals, as measured by ELISA. Therefore, these data suggest that the production of IL-17 in MK-886-treated, immunized mice could contribute to the generation of a protective immune response after infection with $M$. tuberculosis.
\end{abstract}

Key words: Tuberculosis; Prime-boost immunization; Leukotrienes

\section{Introduction}

Tuberculosis (TB) is a disease caused by infection with Mycobacterium tuberculosis. The World Health Organization estimates that 2-3 million people die from TB each year, and one third of the planet's population is infected with the tubercle bacillus. However, almost $90 \%$ of those infected do not develop the disease. Conditions affecting the immune system, such as co-infection with HIV, diabetes or malnutrition, increase the risk of developing the active form of the disease (1).

Cell-mediated immune responses play a critical role in the host's defense against intracellular pathogens such as M. tuberculosis. Protection is associated with the development of interferon-gamma (IFN-y)-producing Th1 CD4+ cells, which activate macrophage microbicidal mechanisms against intracellular bacteria. Activated macrophage also secretes tumor necrosis factor-alpha (TNF- $\alpha$ ) and

Correspondence: V.L.D. Bonato, Departamento de Bioquímica e Imunologia, Faculdade de Medicina de Ribeirão Preto, Av. Bandeirantes, 3900, 14049-900 Ribeirão Preto, SP, Brasil. Fax: +55-16-3633-6840. E-mail: vlbonato@fmrp.usp.br

Received January 28, 2010. Accepted May 24, 2010. Available online June 7, 2010. Published July 9, 2010. 
chemokines, such as monocyte chemotactic protein-1, macrophage inflammatory protein-1 $\alpha$ (MIP-1 $\alpha)$, MIP-1 $\beta$ and RANTES (regulated upon activation, normal $T$ cell expressed and secreted), which plays a key role in granuloma formation (reviewed in Ref. 2). It has been reported that cytotoxic CD8+ T cell-mediated killing of infected host cells and IFN-y-producing CD8+ T cells also play a role in the defense against $M$. tuberculosis (3). In addition to IFN- $Y$ and cytokines such as interleukin-12 (IL-12) and TNF- $\alpha(4,5)$, lipid mediators, such as leukotrienes (LT), participate in the host defense against $M$. tuberculosis infection. M. tuberculosis-infected mice treated with the inhibitor of LT synthesis, MK-886, showed an increase in lung bacterial burden and a decrease in IFN- $\mathrm{Y}, \mathrm{IL}-12$ and nitrite secretion. In addition, the influx of neutrophils and mononuclear cells to the lungs of MK-886-treated mice was reduced (6).

Since LT contribute to IL-12 production, these lipid mediators may indirectly participate in CD4+ T differentiation to the Th1 pattern. Furthermore, during a secondary immune response against infection by Histoplasma capsulatum, LT play a role by recruiting memory CD4+ and CD8+ cells to the lung (7). The activity of LT as powerful leukotropic and proinflammatory mediators involved in host defenses in a variety of infectious diseases $(6,8,9)$, including mycobacterial infections (6), led us to suggest that LT might be involved in the protective immune response generated by heterologous prime-boost immunization with Bacille Calmette-Guérin (BCG) prime/DNA-HSP65 booster against M. tuberculosis infection.

BCG is still the only vaccine available for TB prevention. In attempts to develop a new vaccine against $T B$, some groups have focused on developing recombinant antigens, while others have concentrated their efforts on the development of a recombinant BCG vaccine capable of inducing greater protective immunity than conventional BCG (10). A different prophylactic vaccination strategy consists of boosting the immunogenicity of the BCG vaccine. This strategy is defined as prime-boost immunization. Secreted antigens, such as Mtb72F, 85A, or 85B antigens, and the 6-kDa early-secretion antigen target (ESAT-6) have been shown to be promising candidates for BCG-boosting vaccines in mice, guinea pigs, and nonhuman primates (11-13). Experimental data collected by our group over the last few years have shown that a DNA vaccine encoding the $M$. leprae $65-\mathrm{kDa}$ heat shock protein gene (DNA-HSP65) represents a strategy for experimental TB prevention (14). In an attempt to optimize the protective effect of this DNA vaccine and to boost BCG immunization, we found that heterologous prime-boost immunization using BCG priming/DNA-HSP65 boosting conferred significant protection to challenged mice (15).

In the present study, we confirmed that LT contribute to the protective immune response against $M$. tuberculosis infection in MK-886-treated mice, which were more susceptible to M. tuberculosis infection. However, LT were not essential in prime-boost vaccinated mice treated with MK-886, which remain protected after challenge with $M$. tuberculosis.

\section{Material and Methods}

\section{Mice}

Female 6-8-week-old BALB/c mice were obtained from the Faculdade de Medicina de Ribeirão Preto (Universidade de São Paulo, Brazil). Mice were housed under barrier conditions in a level III biohazard laboratory and provided with food and sterile water. Experiments were conducted according to the Comissão de Ética em Experimentação Animal da Faculdade de Medicina de Ribeirão Preto (CETEA) ethical guidelines.

\section{Bacteria}

M. tuberculosis H37Rv strain (\#27294; ATCC, USA) was grown for 7 days at $37^{\circ} \mathrm{C}$ in $7 \mathrm{H} 9$ Middlebrook broth (Difco, USA) enriched with $0.2 \%(\mathrm{v} / \mathrm{v})$ glycerol and $10 \%$ $(\mathrm{v} / \mathrm{v})$ oleic acid albumin dextrose (Difco) prepared as previously described (15). M. bovis BCG (Moreau strain) was purchased from Fundação Ataulpho Paiva (FAP), Rio de Janeiro, RJ, Brazil.

\section{Plasmid construction}

pVAX-HSP65 (DNA-HSP65) vaccine was constructed from the pVAX vector (Invitrogen, USA) as described $(14,15)$. Endotoxin concentration was determined using the Limulus amebocyte lysate kit QCL-1000 (BioWhittaker, USA). The amount of endotoxin used for the plasmid in this study was $\leq 0.1$ endotoxin units/ $\mu$ g DNA.

\section{Immunization and challenge infection}

For heterologous vaccination, each animal received 1 $x 10^{5} \mathrm{M}$. bovis BCG in $100 \mu \mathrm{L}$ phosphate-buffered saline (prime) by the subcutaneous route. After 15 days, animals received $50 \mu \mathrm{g}$ DNA-HSP65 in $50 \mu \mathrm{L}$ saline plus $50 \%$ sucrose into each quadriceps muscle (booster). Thirty days after the last immunization, mice were challenged by injection of $M$. tuberculosis ( $1 \times 10^{5}$ bacteria/animal) by the intratracheal route (15).

\section{Treatment of mice with MK-886}

To inhibit endogenous LT production, animals were treated by gavage with the MK-886 compound (Merck Frosst, Canada) $(5 \mathrm{mg} / \mathrm{kg}$ ) diluted in $0.5 \mathrm{~mL}$ water $1 \mathrm{~h}$ prior to infection with $M$. tuberculosis, repeated every $24 \mathrm{~h}$ for 30 days. A dose of $5 \mathrm{mg} \cdot \mathrm{kg}^{-1}$. day ${ }^{-1}$ has been shown to inhibit LT synthesis in a model of pulmonary histoplasmosis (8). Control groups were treated with water.

\section{Colony-forming units assay}

The lower right and middle lobes of the lungs were digested with $0.5 \mu \mathrm{g} / \mathrm{mL}$ Liberase Blendzyme 2 solution 
(Roche, USA) and $25 \mathrm{U} / \mathrm{mL}$ DNAse (Sigma, USA), as previously described $(14,15)$. For the colony-forming units (CFU) assay, serial dilutions of digested lungs were plated onto 7H11 agar medium (Difco Laboratories, USA) and CFU number was determined 28 days after incubation at $37^{\circ} \mathrm{C}$.

\section{Cytokine production}

The left lobes of the lungs were removed and homogenized (Mixer Homogenizer, Labortechnik, Germany) in 2 $\mathrm{mL}$ RPMI 1640 (Sigma), centrifuged at $1500 \mathrm{~g}$ and stored at $-70^{\circ} \mathrm{C}$ until assayed. IFN-Y and IL-12 concentrations were determined in the supernatants by ELISA according to manufacturer instructions. The following anti-mouse purified monoclonal antibodies (mAb) were used $(1 \mu \mathrm{g} /$ $\mathrm{mL}$ ): IFN-Y (R4-6A2) and IL-12 (C15.6; BD BiosciencesPharMingen, USA). Cytokine-antibody complexes were detected via the addition of $0.5 \mu \mathrm{g} / \mathrm{mL}$ of the anti-mouse biotinylated mAb IFN-y (XMG1.2) and IL-12 (C17.8; BD Biosciences-PharMingen). For IL-17 detection, ELISASET R\&D was used.

\section{Histology}

For histopathological analysis, the right upper lobes of the lungs were fixed in $10 \%$ formalin, embedded and processed in paraffin blocks, and then sectioned for light microscopy. Five-micron thick sections were stained with hematoxylin-eosin. All samples were analyzed by a pathologist in a double-blind assay.

\section{Statistical analysis}

All data are reported as means \pm SEM. Data were compared using the PRISM software (version 5.0, GraphPad, USA). Variables among groups were compared by analysis of variance (ANOVA) followed by the Tukey test. Values of $\mathrm{P}<0.05$ were considered to be significant.

\section{Results}

\section{LT are not essential for the protection conferred by prime-boost immunization}

To investigate the role of LT in the protection conferred by heterologous BCG/DNA-HSP65 (prime-boost) immunization against $M$. tuberculosis infection, we evaluated the bacterial clearance in the lungs of immunized mice treated with MK-886 and challenged with $M$. tuberculosis in comparison to the lungs of non-immunized (saline) infected and treated mice. Figure $1 \mathrm{~B}$ shows that the treatment with MK-886 of non-immunized mice increased their susceptibility to infection when compared to water-treated mice ( $P$ $<0.05)$. As expected, immunization using the prime-boost strategy resulted in a significant reduction in CFU numbers in the lungs of water-treated $M$. tuberculosis-challenged mice when compared to the lungs of non-immunized and infected mice. However, when compared to animals immu- nized by prime-boost strategy and treated with MK-886, no differences in CFU numbers was observed. These results suggest that prime-boost immunization was efficient in protecting mice against the $M$. tuberculosis challenge even in the absence of endogenous LT. Taken together, these results suggest that LT are important for the immune response against TB, but are not an essential component of the protective immune response induced after prime-boost heterologous immunization.

Regarding the production of cytokines, infected mice treated with MK-886 secreted lower concentrations of IFN-Y and IL-12 compared to infected mice treated with water $(P$ $>0.05$; Figure $1 \mathrm{C}$ and $\mathrm{D}$, respectively). Prime-boost im-
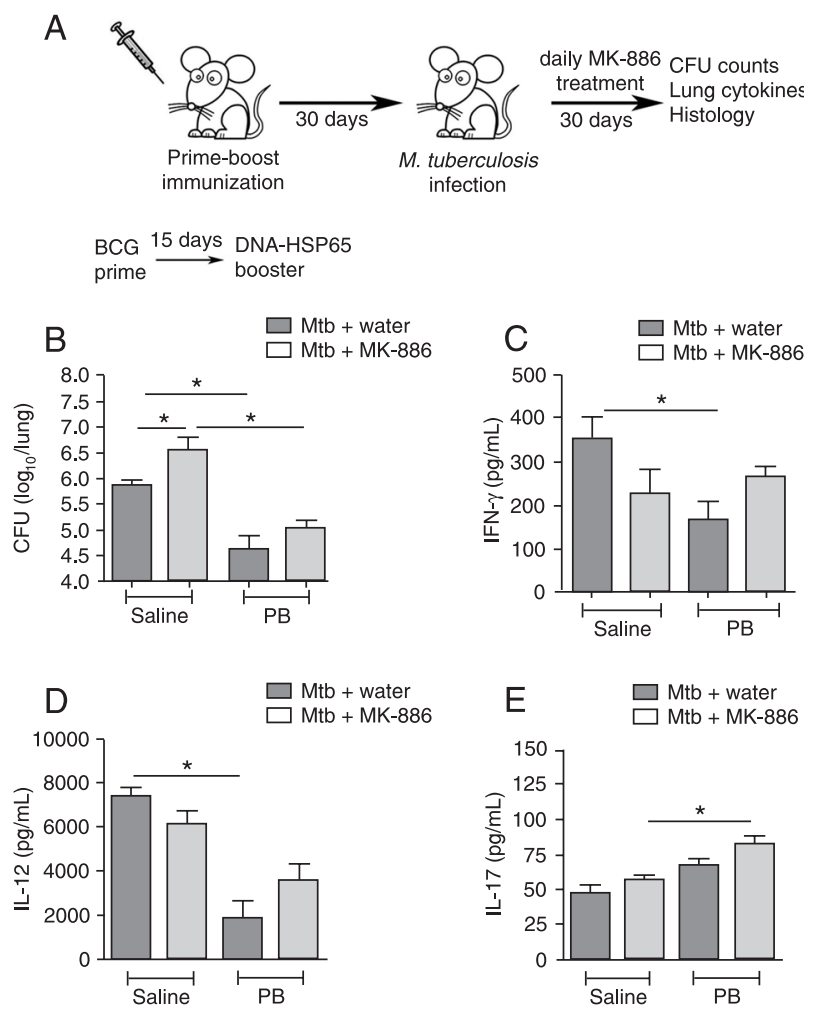

Figure 1. Effect of the inhibition of endogenous leukotriene (LT) synthesis on Mycobacterium tuberculosis growth and cytokine production in lungs of prime-boost-immunized, $M$. tuberculosischallenged mice. Animals were immunized by the prime-boost (PB) strategy and challenged with $10^{5} \mathrm{M}$. tuberculosis bacilli (Mtb) by intratracheal administration 30 days after the immunization schedule. Saline-injected animals were used as controls. One hour before and every $24 \mathrm{~h}$ after infection, animals were treated by gavage with water or MK-886 (5 mg $\mathrm{kg}^{-1} \cdot$ day $\left.^{-1}\right)(\mathrm{A})$. Thirty days after challenge, the lung bacterial load was evaluated by colony-forming unit (CFU) counting (B). The production of interferon-gamma (IFN-Y) (C), interleukin -12 (IL-12) (D), and IL-17 (E) was evaluated in lung homogenates by ELISA. Data are reported as means \pm SEM ( $N=6-11$ for CFU counting and 4-9 for the measurement of cytokines). ${ }^{*} \mathrm{P}<0.05$ (ANOVA followed by the Tukey test). 
munization of water-treated mice reduced IFN-y and IL-12 production compared to the respective non-immunized group $(P<0.05)$. There were no significant differences in IFN-Y and IL-12 levels between mice immunized by the prime-boost strategy and treated with MK-886 and those immunized and treated with water $(P>0.05)$. Moreover, immunized, challenged and MK-886-treated mice also secreted lower concentrations of IFN- $\gamma$ and IL-12 compared to non-immunized, infected mice treated with MK-886 (Figure $1 \mathrm{C}$ and $\mathrm{D})$. Only those groups immunized by the prime-boost strategy and treated with MK-886 showed an increase in IL-17 concentrations compared to non-immunized, MK886-treated mice $(P<0.05$; Figure 1E).

\section{Inhibition of LT synthesis impaired leukocyte migration to the lungs of $M$. tuberculosis-infected mice}

Given the fact that LT were first characterized for their role in leukocyte recruitment, we next evaluated inflammatory cell influx to the lungs. Figure 2 shows that infected mice $\left(10^{5} \mathrm{M}\right.$. tuberculosis bacilli) treated with water (Mtb +
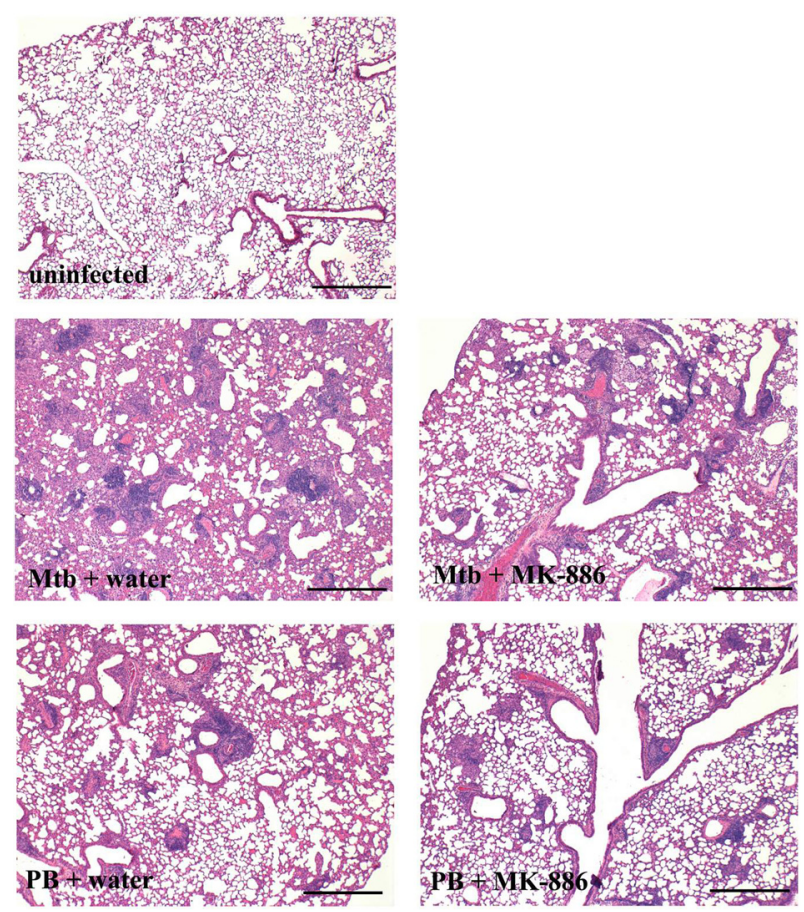

Figure 2. Cell influx into the lungs of prime-boost-immunized, $M y$ cobacterium tuberculosis-challenged mice treated with MK-886. Experimental groups were treated, immunized and challenged as described in the legend to Figure 1A. Representative HE-stained sections of lungs of uninfected mice (control), animals infected with $M$. tuberculosis and treated with MK-886 (Mtb + MK-886) or water (Mtb + water), and lungs of animals immunized with primeboost (PB), challenged with $M$. tuberculosis and treated with MK886 (PB + MK-886) or with water (PB + water) are shown. Original magnification 50X. Bar $=500 \mu \mathrm{M}$. water) had an intense lung parenchyma injury represented by diffuse, extensive leukocyte infiltration and bronchusassociated lymphoid tissue hyperplasia compared to uninfected mice. Infected mice treated with MK-886 (Mtb + MK-886) had more visibly preserved lung areas, which showed a lower level of leukocyte recruitment than that observed in infected, water-treated mice. Lungs of primeboost-immunized, water-treated mice (PB + water) also showed a reduced cell recruitment compared to lungs of non-immunized infected mice (Mtb + water). The lungs of prime-boost immunized, MK-886-treated mice (PB + MK886) exhibited a considerably lower cell influx in comparison to lungs of prime-boost-immunized, water-treated mice. These histology results confirm the importance of LT in leukocyte recruitment to the lungs during $M$. tuberculosis infection.

\section{Discussion}

In the present study, we confirmed the results of Peres et al. (6), who provided evidence for the importance of LT in the immune response against $M$. tuberculosis infection. We also confirmed that mice treated with MK-886 were more susceptible to $M$. tuberculosis infection. This higher susceptibility was associated with the impaired influx of leukocytes to the lungs after infection and with decreased concentrations of IFN- $\gamma$ in the lungs, as previously reported by Peres et al. (6). Our results, together with those reported by Peres et al. (6), suggest that LT can modulate soluble factors related to protection, such as IFN- $\gamma$ and nitrite, and can stimulate the recruitment of leukocytes to the site of infection. In a similar manner, treatment of BALB/c mice with MK-886 increased their susceptibility to infection with $H$. capsulatum (8). On the other hand, Bafica et al. (16) demonstrated that 5-lipoxygenase (5-LO-/-) knockout mice were more resistant to $M$. tuberculosis infection than wild-type mice. These investigators attributed the better ability of 5-LO-/- mice to control infection to a decreased synthesis of lipoxin A4, a 5-LO-derived anti-inflammatory mediator (17). However, Peres et al. (6) also showed a decrease in the production of lipoxin A4 in MK-886-treated infected BALB/c mice. The discrepancy between our results and those of Bafica et al. (16) may be related to the different route of infection (intratracheal vs aerosol) and to the inoculum size ( $10^{5}$ bacilli vs 50 or 300 bacilli) because $M$. tuberculosis infection can produce a variety of outcomes, including slowly progressive TB or chronic experimental disease according to the route of infection, inoculum size and mouse strain (18).

Although our results show that LT are related to an effective immune response against $M$. tuberculosis infection, we found that these lipid mediators do not seem to have a key role in the effector immune response induced by a prime-boost heterologous vaccination against experimental TB. The protection conferred by prime-boost immunization 
against a challenge with $M$. tuberculosis was similar for MK-886-treated and -untreated mice. In addition, the lung parenchyma from prime-boost-immunized, MK-886-treated mice showed better preserved lung areas than that of from prime-boost-immunized, untreated mice.

Since we did not observe a significant difference in IFN- $\mathrm{Y}$ and an increase in IL-17 production in lungs of prime-boost-immunized, MK-886-treated mice compared to non-immunized, MK-886-treated mice, we suggest that the induction of Th17 cells during immunization, and consequently the production of IL-17 by effector and/or memory cells during infection, could play a role in the protection maintained after treatment with MK-886. IL-17 has been described as a potent proinflammatory cytokine, which is able to induce recruitment of neutrophils (19). It has been recently demonstrated that Th17 cells are essential for the protection elicited by a vaccine against TB (ESAT-6 peptide mixed with DDA, MPL and TDM adjuvants). The protection was based on the production of IL-23, which induced the differentiation of Th17 cells. This cell population triggered chemokine production with subsequent recruitment of Th1 cells to the lungs (20). In addition, results from our group also suggest that IL-17 is related to protection against experimental and human TB (Paula MO and Wowk PF, unpublished data). These data suggest that prime-boost immunization was able to induce differentiation of Th17 cells, and in the

\section{References}

1. WHO. Global tuberculosis control. Geneva: World Health Organization; 2008.

2. Algood HM, Chan J, Flynn JL. Chemokines and tuberculosis. Cytokine Growth Factor Rev 2003; 14: 467-477.

3. Silva CL, Bonato VL, Lima KM, Coelho-Castelo AA, Faccioli LH, Sartori A, et al. Cytotoxic T cells and mycobacteria. FEMS Microbiol Lett 2001; 197: 11-18.

4. Flynn JL, Goldstein MM, Chan J, Triebold KJ, Pfeffer K, Lowenstein $\mathrm{CJ}$, et al. Tumor necrosis factor-alpha is required in the protective immune response against Mycobacterium tuberculosis in mice. Immunity 1995; 2: 561-572.

5. Flynn JL, Goldstein MM, Triebold KJ, Sypek J, Wolf S, Bloom BR. IL-12 increases resistance of BALB/c mice to Mycobacterium tuberculosis infection. J Immunol 1995; 155: 2515-2524.

6. Peres CM, de Paula L, Medeiros AI, Sorgi CA, Soares EG, Carlos D, et al. Inhibition of leukotriene biosynthesis abrogates the host control of Mycobacterium tuberculosis. Microbes Infect 2007; 9: 483-489.

7. Medeiros AI, Sa-Nunes A, Turato WM, Secatto A, Frantz FG, Sorgi CA, et al. Leukotrienes are potent adjuvant during fungal infection: effects on memory T cells. J Immunol 2008; 181: 8544-8551.

8. Medeiros Al, Sa-Nunes A, Soares EG, Peres CM, Silva CL, Faccioli LH. Blockade of endogenous leukotrienes exacerbates pulmonary histoplasmosis. Infect Immun 2004; 72 : 1637-1644.

9. Peters-Golden M, Canetti C, Mancuso P, Coffey MJ. Leu- deficiency in LT synthesis, IL-17 may compensate for the low levels of this lipid mediator by contributing to leukocyte recruitment for the control of infection. To confirm the role of IL-17 in the protection induced by prime-boost immunization in LT absence, experiments using IL-17 knockout mice remain to be explored.

Overall, the present results confirm the essential role of $L T$ in the immune response against $M$. tuberculosis. Besides, the immunization using the prime-boost strategy seems to induce inflammatory mediators, such as IL-17, which probably contribute to the protective efficacy, even with an inhibition of LT production.

\section{Acknowledgments}

We would like to thank Ana Paula Masson Soares and Izaíra Tincani Brandão, from Núcleo de Pesquisas em Tuberculose, Departamento de Bioquímica e Imunologia da Faculdade de Medicina de Ribeirão Preto, for technical assistance and Elaine Medeiros Floriano, from Departamento de Patologia da Faculdade de Medicina de Ribeirão Preto, for histological assistance. Research supported by FAPESP (\#07/02407-0), CAPES-PROEX and Núcleo de Pesquisas em Tuberculose (NPT). We are grateful to Merck Frosst, Canada, for furnishing the MK-886. kotrienes: underappreciated mediators of innate immune responses. J Immunol 2005; 174: 589-594.

10. Skeiky YA, Sadoff JC. Advances in tuberculosis vaccine strategies. Nat Rev Microbiol 2006; 4: 469-476.

11. Brandt L, Skeiky YA, Alderson MR, Lobet $Y$, Dalemans W, Turner OC, et al. The protective effect of the Mycobacterium bovis BCG vaccine is increased by coadministration with the Mycobacterium tuberculosis 72-kilodalton fusion polyprotein Mtb72F in M. tuberculosis-infected guinea pigs. Infect Immun 2004; 72: 6622-6632.

12. Dietrich J, Andersen $C$, Rappuoli R, Doherty TM, Jensen CG, Andersen P. Mucosal administration of Ag85B-ESAT-6 protects against infection with Mycobacterium tuberculosis and boosts prior bacillus Calmette-Guerin immunity. $\mathrm{J} / \mathrm{m}$ munol 2006; 177: 6353-6360.

13. McShane H, Pathan AA, Sander CR, Goonetilleke NP, Fletcher HA, Hill AV. Boosting BCG with MVA85A: the first candidate subunit vaccine for tuberculosis in clinical trials. Tuberculosis 2005; 85: 47-52.

14. Bonato VL, Lima VM, Tascon RE, Lowrie DB, Silva CL. Identification and characterization of protective T cells in hsp65 DNA-vaccinated and Mycobacterium tuberculosis-infected mice. Infect Immun 1998; 66: 169-175.

15. Goncalves ED, Bonato VL, da Fonseca DM, Soares EG, Brandao IT, Soares AP, et al. Improve protective efficacy of a TB DNA-HSP65 vaccine by BCG priming. Genet Vaccines Ther 2007; 5: 7.

16. Bafica A, Scanga CA, Serhan C, Machado F, White S, Sher 
A, et al. Host control of Mycobacterium tuberculosis is regulated by 5-lipoxygenase-dependent lipoxin production. J Clin Invest 2005; 115: 1601-1606.

17. Machado FS, Aliberti J. Impact of lipoxin-mediated regulation on immune response to infectious disease. Immunol Res 2006; 35: 209-218.

18. Rook GA, Hernandez-Pando R, Zumla A. Tuberculosis due to high-dose challenge in partially immune individuals: a problem for vaccination? J Infect Dis 2009; 199: 613-618.

19. Korn T, Bettelli E, Oukka M, Kuchroo VK. IL-17 and Th17 cells. Annu Rev Immunol 2009; 27: 485-517.

20. Khader SA, Bell GK, Pearl JE, Fountain JJ, Rangel-Moreno $\mathrm{J}$, Cilley GE, et al. IL-23 and IL-17 in the establishment of protective pulmonary $\mathrm{CD} 4+\mathrm{T}$ cell responses after vaccination and during Mycobacterium tuberculosis challenge. Nat Immunol 2007; 8: 369-377. 